\title{
A Novel Approach to Support the Visual Exploration and Management of Heterogeneous Home Area Network Devices and Services
}

\author{
Yuqian Song, Owen Conlan \\ Trinity College Dublin, \\ Dublin 2, Ireland \\ \{yuqians, owen.conlan\}@cs.tcd.ie
}

\begin{abstract}
With the advance of home networking technologies, people now enjoy increased communications convenience whilst occasionally suffering annoyance from trivial issues. The automated application of appropriate expertise can support ordinary users in managing and diagnosing their own network, despite being unfamiliar with the basic technologies. This presents a significant opportunity to increase user satisfaction and reduce support costs. As a part of my $\mathrm{PhD}$ research, this paper proposes an expert knowledge derived approach to manage, diagnose and resolve network problems in real time through a user-friendly visual interface derived from semantically annotated heterogeneous raw network monitoring data. This approach has been implemented in an early prototype - Home Area Network Monitoring System (HANMS). A detailed description of the current stage of this research and future work is addressed in this paper.
\end{abstract}

Keywords: Home Area Network; HAN; network monitoring; domain ontology; semantic visualization; semantic annotation; network management

\section{Introduction}

As ICT (Information and communications technology) complexity increases, inexperienced users are continuously challenged and perplexed with even simple management and maintenance tasks. In a Home Area Network (HAN) scenario, normal HAN users are often puzzled by trivial wireless network issues. For example: a laptop suddenly suffers a quality degradation of the network connection, perhaps caused by a neighbor initiating some WiFi activity in the same channel - causing interference, or a signal weakens when the antenna of the wireless device is obstructed, or the laptop user may upload a large file which causes network congestion. Such events seem unremarkable but will impact on the end-user's perception of the quality of the service provided by the wireless network connection. Moreover, most users will not have the technical expertise to diagnose these problems in real time. Conversely, if the user could easily diagnose and solve these (often simple) problems, this would avoid unnecessary contact with support staff and reduce the operating costs for both the network provider and HAN users. To address this 
challenge, a novel approach is proposed to bridge the gap between the hard-learned knowledge of domain experts and the cognitive competencies of normal users in a HAN context. This approach aims to achieve the following objectives: to semantically enrich and correlate raw network log data from heterogeneous HAN devices and services with domain knowledge; to detect and analyze anomalies through a real-time diagnosis loop monitoring the constantly evolving HAN devices and services; to appropriately present the highly abstracted, correlated and annotated monitoring data for normal HAN users in a user-friendly visual interface; and finally, to leverage the high-level annotated monitoring data to perform high-level (but groundable) management actions. Related research already addressing some of these objectives will be briefly reviewed in the Related Work section. The Solution Overview \& Evaluation section presents an early prototype of the Home Area Network Monitoring System (HANMS), followed by preliminary evaluation results. Future work and conclusions are described in the last section.

\section{Related Work}

One problem in the rapidly evolving smart home network management area is how to deal with the vast number of dynamic network log datasets. Driven by Semantic Web technologies, Semantic Annotation can be applied to transform and enrich the data and information with formal semantic meanings. In Handschuh and Staab's book [1], a broad range of technologies and methods is introduced for the explicit construction of semantic annotations, including approaches for data extraction, collaboration and translation into Semantic Web metadata. In particular, goal-oriented data extraction is valuable for network $\log$ data annotation, especially for monitoring and management purposes. By facing the challenges of evolution and heterogeneity of HAN technologies and varying user requirements in the complex HAN environment, Brennan et al. [2] presented a policy-based federated service management architecture that addresses these concerns for digital home devices participating in end-to-end communications services, while Jennings et al. [3] proposed an autonomic network management scheme, which allows a networked system to self-govern its behavior within the constraints of the human-specified goals. Another effective strategy is to perform network management by involving ontology-based modeling and reasoning. Hoag [4] presented an approach to apply semantic reasoning techniques for network management and resource allocation to avoid overbuilding and improve quality. Visualization is an effective strategy to align complex data by capitalizing on several human perception capabilities, and a novel interactive visualization system [6] was proposed as an approach to monitor the data collected from a home router and control bandwidth usage for family users. An interactive network management system, called Eden [7], delivered a simple conceptual model to help users understand key aspects of the network by eliminating the barrier and cost of the network technical minutia. Our research is also based on Conlan et al.'s work [9] to express a semantically enriched visualization approch for non-expert users to monitor networks and services. Our work intends to combine the advantages of these research to propose a novel approach for HAN monitoing and management. 


\section{Solution Overview and Evaluation}

This section describes a novel approach for HAN monitoring and management (Figure 1) established in our prototype, HANMS [5].

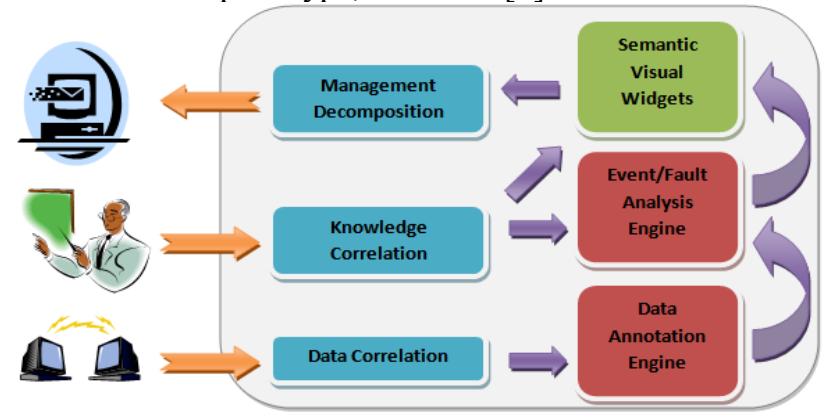

Fig. 1. HAN Monitoring and Management

In this figure, the data annotation process is implemented in a layered annotation engine to enhance large amounts of heterogeneous data with multi-level discrete semantic encodings according to the embedded network expertise to support the highlevel processing. In the current HANMS prototype, the raw wireless log data are gathered from diverse wireless devices in a HAN test bed; expert domain knowledge is modeled into domain ontologies; and the annotation is applied in a two-levelannotation process. Through the Event/Fault Analysis Engine, real-time semantically enhanced data is observed in an analysis and diagnosis loop according to correlated expertise, in HANMS, modeled in network event ontologies. Using HANMS visual widgets (Figure 2), detected events/faults, and network information, is automatically and intelligently presented and highlighted. Management requests will be expressed through high-level management actions and these actions will be decomposited into lower level actions [8] to directly affect or control HAN services and devices according to expert defined ontologies and rules. The management decomposition is not supported by HANMS at this stage.

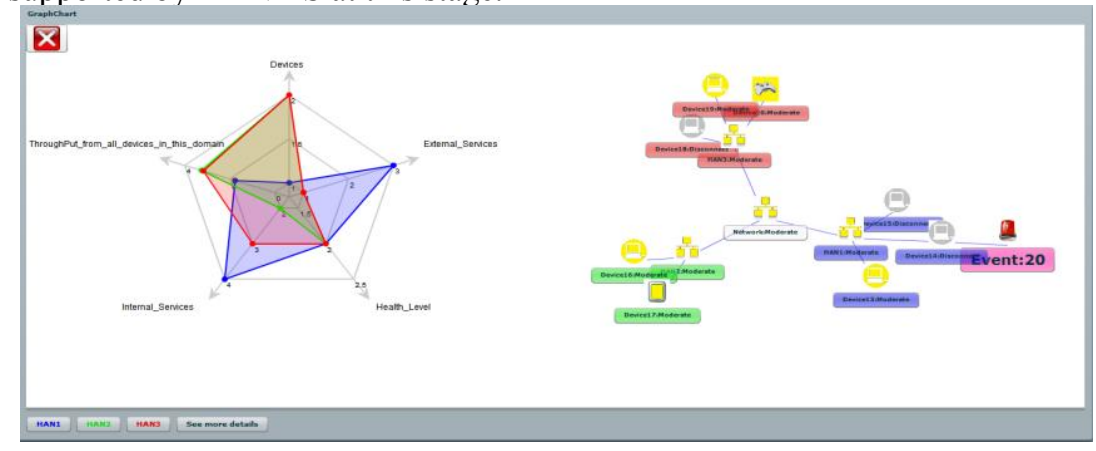

Fig. 2. HANMS prototype screenshot

The current HANMS prototype has been deployed and initial evaluation was carried out with two target groups: normal HAN users and domain experts. The majority of feedback (more than 70\%) was positive, especially in the usability and UI design whilst identified limitations will be addressed in the future development. 


\section{$4 \quad$ Future Work and Conclusions}

The current HANMS prototype is still under active development. For high-level network management, multi-resource HAN monitoring merits further development. As a part of the FAME project, HANMS has the potential to integrate with a number of prototype systems, e.g. smart home composite UPnP services. By consuming the knowledge models of UPnP events and faults [2], HANMS could present a more effective and efficient approach to diagnose the real time event/fault with high complexity. Based on this integration, high-level management goals can be decomposed into enforceable UPnP actions to control low-level devices and services. According to the up-to-date HANMS prototype deployment and evaluation results, this approach will assist ordinary end users to diagnose and resolve potential problems in a HAN, which presents a significant opportunity to reduce support costs and increase user satisfaction. This work will also show that it is possible to alleviate the difficulties of non-expert users in HAN scenarios by combining Semantic Web and Visualization technologies, while capturing and integrating domain experts' insights.

Acknowledgments This work is partly funded by Science Foundation Ireland via grant 08/SRC/I1403 - Federated, Autonomic Management of End-to-End Communications Services (FAME).

\section{References}

1. Handschuh, S., Staab, S.: Annotation for the semantic web. IOS Press (2003)

2. Brennan, R., Lewis, D., Keeney, J., Etzioni, Z., Feeney, K., O'sullivan, D., Lozano, J., and Jennings, B.: Policy-based integration of multiprovider digital home services, In: IEEE Network, vol. 23, pp. 50-56. IEEE Press. (2009)

3. Jennings, B., et al.: Towards autonomic management of communications networks, In: IEEE Communications Magazine, vol. 45, pp. 112-121. IEEE Press (2009)

4. Hoag, J.C., Hayes-Roth, F.A.: Semantic Reasoning for Adaptive Management of Telecommunications Networks, In: IEEE International Conference on Systems, Man and Cybernetics, Taipei, Taiwan (2006)

5. Song, Y., et al.: An Ontology-driven Approach to support Wireless Network Monitoring for Home Area Networks, In: 7th International Conference on Network and Service Management (CNSM 2011).Submitted. (2011)

6. Chetty, M.,et al.: Who' s Hogging The Bandwidth: The Consequences Of Revealing The Invisible In The Home, In: 28th international conference on Human factors in computing systems, New York. (2010)

7. Yang, J., Edwards, W.K., Haslem, D.: Eden: supporting home network management through interactive visual tools, In: 23nd annual ACM symposium on User interface software and technology (UIST '10). ACM, pp 109-118. (2010)

8. Keeney, J., et al.: Towards the use of Policy Decomposition for Composite Service Management by Non-expert Endusers, In: the 5th Network Operations and Management Symposium Workshops (NOMS Workshops), IEEE/IFIP, IEEE, pp 163-164. (2010)

9. Conlan, O., Keeney, J., Hampson, C., Williams, F.P., "Towards Non-expert Users Monitoring Networks and Services through Semantically Enhanced Visualizations" to appear in Proceedings of the 6th International Conference on Network and Service Management (CNSM 2010), Niagara Falls, Canada (2010) 